\section{Resignificación de la extensión a 100 años de la Reforma Universitaria de 1918}

Gustavo Menéndez

Secretario de Extensión de la Universidad

Nacional del Litoral, Argentina.

Coordinador de la Comisión Permanente

de Extensión Asociación de Universidades

del Grupo Montevideo.

gmenendez@unl.edu.ar
A 100 años de la Reforma Universitaria de 1918 /

Perspectivas

RECEPCIÓN: 29/06/17

ACEPTACIÓN FINAL: 24/09/17

\section{Resumen}

Este artículo pretende reflexionar sobre los sentidos que ha adquirido la extensión universitaria en el contexto latinoamericano en las proximidades del centenario de la Reforma Universitaria. Así, un abanico de preguntas orienta las reflexiones: ¿qué ha significado este hecho para las instituciones de educación superior latinoamericanas y caribeñas? ¿Qué aspectos se mantienen vigentes y cuáles se han resignificado con el tiempo? ¿Cuál ha sido el aporte de la extensión en el desarrollo de las instituciones universitarias? ¿Qué ha pasado con el conocimiento y de qué manera se plantea la integración de las funciones sustantivas? A su vez, el texto repasa la densidad conceptual y el entramado teórico sobre el que puede pensarse la extensión universitaria en la actualidad y presenta nuevos interrogantes que permiten reflexionar sobre modelos universitarios: ¿cómo se manifiestan y resuelven las tensiones entre calidad, pertinencia-misión social, autonomía, pensamiento crítico y pleno ejercicio democrático? ¿Cómo se proyecta la universidad reformista hacia las próximas décadas? ¿De qué manera ponderamos el rol de la extensión universitaria en el presente? ¿Cómo nos imaginamos o proyectamos la extensión para el futuro?

\section{Palabras claves}

- Reforma Universitaria

- Conceptos de extensión universitaria

- Dimensiones de la extensión

- Agenda de extensión

\section{Resumo}

Este artigo tenta refletir sobre os sentidos que tem adquirido a extensão universitária no contexto latino-americano nas proximidades do centenário da Reforma Universitária. Assim sendo, um leque de perguntas orienta as reflexões: O que significou este fato para as instituições de educação superior latino-americanas e caribenhas? Que aspectos se mantêm vigentes e quais se têm ressignificado com o tempo? Qual foi a contribuição da extensão no desenvolvimento das instituições universitárias? O que aconteceu com o conhecimento e de que forma se expõe a integração das funções substantivas? Por sua vez, o texto analisa a densidade conceitual e a estrutura teórica na qual se pode pensar a extensão universitária na atualidade e apresenta novas indagações que permitem refletir sobre modelos universitários: Como se manifestam e resolvem as tensões entre qualidade, pertinência-missão social, autonomia, pensamento crítico e exercício pleno de democracia? Como se projeta a universidade reformista para as próximas décadas? De que forma ponderamos o papel da extensão universitária atualmente? Como imaginamos ou projetamos a extensão para o futuro?

Palavras-chave

- Reforma Universitária

- Conceitos de extensão universitária

- Dimensiões da extensão

- Agenda de extensão
Para citación de este artículo

Menéndez, G. (2017). Resignificación de la extensión

a 100 años de la Reforma Universitaria de 1918.

Revista +E versión en línea, 7(7), 24-37. Santa Fe,

Argentina: Ediciones UNL. 


\section{Introducción}

Sin lugar a dudas, uno de los desafíos más importantes en materia de extensión universitaria que afrontan hoy las universidades latinoamericanas y caribeñas, y que se proyecta para los próximos años, está relacionado con el objetivo de lograr una mayor institucionalización y reconocimiento académico de esta función universitaria. Para lograr este reconocimiento es fundamental que la extensión forme parte de la vida académica e institucional de la universidad, que se integre adecuadamente con la investigación y la docencia; que contribuya de manera significativa a una mejor calidad y pertinencia universitaria; que participe en los procesos de enseñanza y aprendizaje; que intervenga en la generación de nuevos conocimientos y contribuya a la apropiación social de los mismos y que sume sus esfuerzos a una mayor inclusión y cohesión social y cultural, especialmente de los sectores más vulnerables de la sociedad. En este sentido, al momento de plantearse la búsqueda de un mayor grado de institucionalización de la extensión, se precisa profundizar en la definición de principios y valores acerca de la educación en general y de la universidad en particular, como también del enfoque teórico-conceptual respecto de esta función sustantiva denominada extensión universitaria. A su vez, se requiere que esta función esencial se encuentre incorporada institucionalmente, no solo en el estatuto de cada universidad sino en sus planes de desarrollo institucional y en sus diferentes reglamentaciones. Por supuesto que en este camino de consolidación de la extensión resulta fundamental que las políticas acordadas en la universidad en materia de extensión universitaria se traduzcan en sus diversos instrumentos de gestión que permitan identificar las asignaciones de recursos, la definición de las prácticas institucionales y la reflexión crítica.

Este es el recorrido institucional que viene siguiendo la extensión en la mayoría de las universidades de la región latinoamericana, especialmente en las universidades públicas que dependen del Estado.

Esta posición respecto de la extensión encuentra su referencia histórica en la Reforma Universitaria de 1918 que se manifiesta en Córdoba, Argentina. Este hito signa un modelo de universidad que asume una concepción democrática, autónoma, crítica y creativa, que considera a la educación como un bien público social y un derecho humano fundamental. Desde este lugar, concibe a la extensión como sinónimo de compromiso social, de inclusión, de diálogo y de democratización de los conocimientos. Así, las políticas de extensión son parte de una agenda compartida entre los actores sociales, el Estado y la comunidad universitaria con profundo sentido académico y de pertinencia social. Estas políticas tienen como horizonte contribuir a la transformación social a partir del reconocimiento de las problemáticas sociales, culturales y productivas de la región en la que se encuentra inserta.

Sin embargo, fue en las últimas décadas que la extensión universitaria adquirió este reconocimiento dentro de las agendas universitarias. Un síntoma de ello es la construcción y el aporte de las redes académicas regionales, tales como la UDUAL (Unión de Universidades de América Latina), AUGM (Asociación de Universidades del Grupo de Montevideo), CSUSCA (Consejo Superior Universitario Centroamericano).

Como mención especial, se destaca además el aporte que viene realizando la ULEU (Unión Latinoamericana de Extensión Universitaria) como el espacio académico institucional de extensión más importante y representativo de las universidades latinoamericanas y caribeñas que se ha tenido en los últimos 20 años, ya que a través de los congresos de extensión que la ULEU ha organizado, de sus declaraciones, exposición de enfoques y experiencias, ha contribuido de manera significativa al desarrollo y proyección de la extensión en las universidades de la región.

A su vez, cabe destacar el crecimiento en materia de extensión en las universidades miembros de AUGM, y con ello el fortalecimiento del espacio de construcción colectiva que representa la Comisión Permanente de Extensión del Grupo, que lleva adelante una nutrida agenda de trabajo que convoca e involucra a las universidades miembros interesadas en participar en este espacio de construcción, reflexión y socialización de enfoques teóricos y experiencias de prácticas de extensión universitaria en la región. A 100 años de la Reforma Universitaria de 1918, nos preguntamos en este artículo: ¿qué ha significado este hecho para las instituciones de educación superior, especialmente para universidades latinoamericanas y caribeñas? ¿Qué aspectos se mantienen vigentes y cuáles se han resignificado con el tiempo? ¿Cuál ha sido el aporte de la extensión en el desarrollo de las instituciones universitarias? ¿Qué ha pasado con el conocimiento y de qué manera se plantea la integración de las funciones sustantivas? ¿Cómo se manifiestan y resuelven las tensiones entre calidad, pertinencia-misión social, autonomía, pensamiento crítico y pleno ejercicio democrático? ¿Cómo se proyecta la universidad reformista hacia las próximas décadas? ¿De qué manera ponderamos el rol de la extensión universitaria en el presente? ¿Cómo nos imaginamos o proyectamos la extensión para el futuro? Para poder responder algunas de estas preguntas resulta indispensable realizar un recorrido histórico, teórico y conceptual 


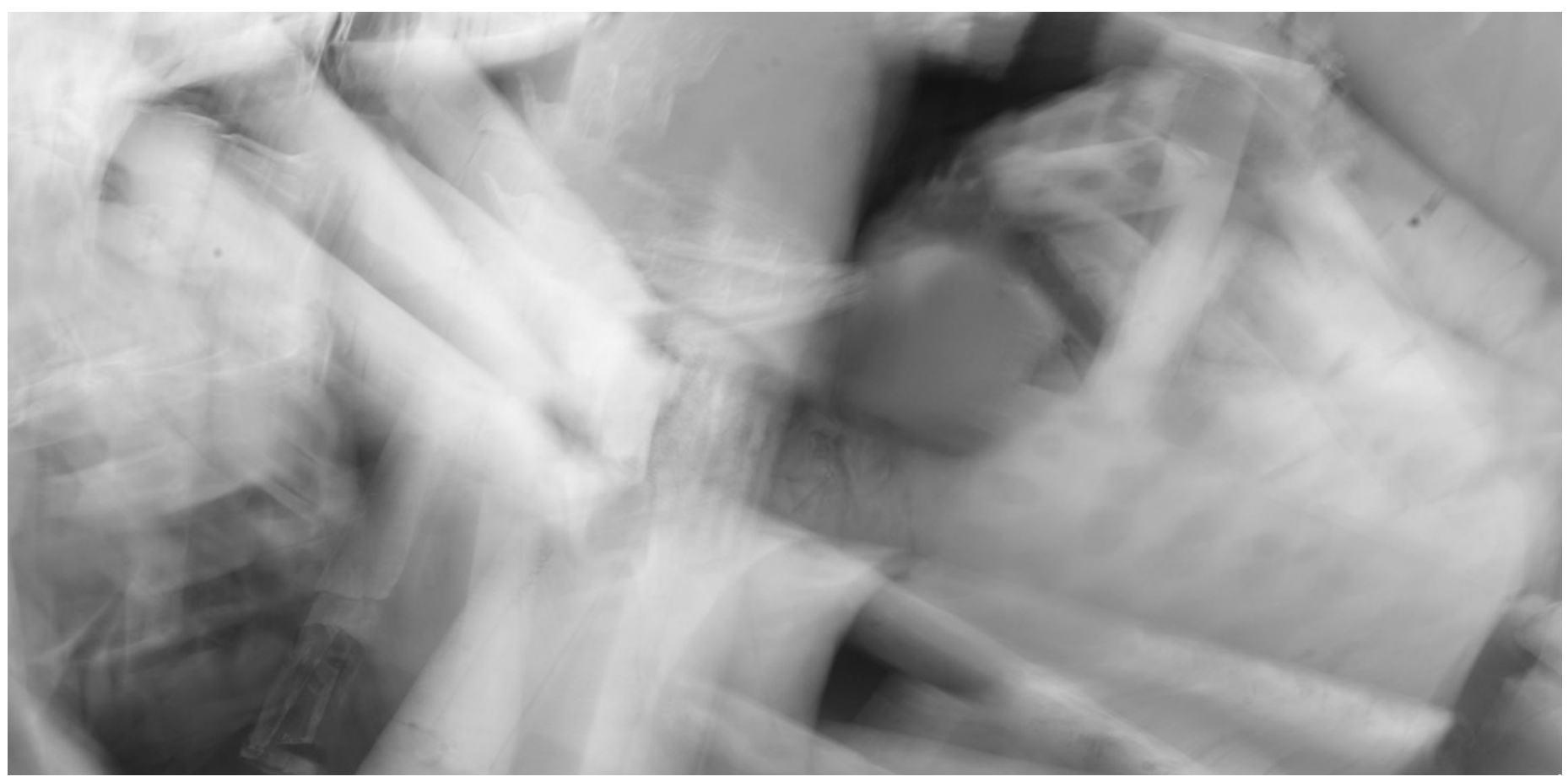

(C) Hugo Pascucci

de la extensión universitaria que nos permita conocer acerca de esta función sustantiva de la universidad, valorar su riqueza multidimensional e identificar los principales aspectos y contenidos que deberían ser tenidos en cuenta a la hora de tomar la extensión como objeto de estudio, debate y proyección.

\section{Extensión y misión social de la universidad}

El concepto de "misión social" de la universidad forma parte de una construcción histórica cuyas referencias iniciales encontramos a partir de las primeras décadas del siglo XIX en las universidades europeas. De manera muy embrionaria y difusa, comenzó a identificarse una misión social de la universidad preocupada por dar respuestas a la población en términos educativos, en general $y$, en particular, vinculada a la capacitación y formación en oficios frente al surgimiento de una nueva clase social como resultado del proceso de la revolución industrial, en pleno desarrollo en la mayoría de los países europeos en ese entonces.

Esta noción fue dándole forma y existencia a la función social de la universidad y luego comenzaría a denominarse "extensión universitaria".

La extensión universitaria tiene su origen histórico en el controvertido siglo XIX, en el que confluye un conjunto de circunstancias que favorecen la aparición de esta función, considerada más tarde esencial por las instituciones universitarias. Sus raíces más profundas están directamente relacionadas con la expansión de los ideales de la Revolución Francesa en el terreno político; la organización del proletariado de clase en cuanto a lo social; las nuevas corrientes de pensamiento en lo filosófico y, por último, en el plano cultural, con la creación de numerosos movimientos artísticos y literarios entre los que se destaca el Romanticismo. Tal como lo plantea Palacios Moroni:

"La extensión, como una de tantas cristalizaciones de la educación, nació cuando la enseñanza aristocrática perdía sus prestigios ante las corrientes sociales, que las defensas de cierta clase y el advenimiento a la vida pública del pueblo produjeron desde el comienzo del siglo (XIX). La educación empezaba a perder en las doctrinas los pujos individualistas, personales, de la Edad Media, y surgían las instituciones populares, las escuelas públicas, las conferencias para adultos, las bibliotecas de vulgarización, que realizaban sus aspiraciones ideales. No viven fuera del mundo real las universidades, y prestaron su concurso, y vertieron en la corriente cuanto estaba de su parte para la función social reflexiva de la ciencia”. (2003:38)

En América Latina, el campo conceptual de la extensión universitaria, asociado al compromiso de la universidad con la sociedad, nació con los postulados de la Reforma Universitaria de 1918 que se manifestó en Córdoba. Estos postulados plantearon la necesidad de participar en la solución de los problemas sociales, económicos 
y políticos, así como en una acción permanente de divulgación de las ciencias y la cultura dirigidas especialmente a los sectores sociales marginados de la enseñanza universitaria.

Si bien esta concepción de universidad presentó alguna relación con la manifestación que venían haciendo diversas universidades europeas en esa época, sin lugar a dudas la Reforma Universitaria le otorgó características distintivas con las que la universidad adquirió un destacado rol a partir de la incorporación de esta función social y cultural bajo el ideal de la democratización del saber y el conocimiento. Este fue en esencia el significado de la extensión para el movimiento reformista de 1918.

En Argentina, la extensión universitaria tuvo sus primeros antecedentes a principios del siglo XX, cuando comenzaron a aparecer diferentes manifestaciones que hacían referencia a este nuevo rol que debían tener las universidades. Uno de estos hechos tuvo lugar en el mismo momento en que se creó la Universidad Nacional de La Plata, en 1905, impulsada por el entonces ministro de Justicia e Instrucción Pública de la Nación Argentina, Dr. Joaquín V. González, quien calificó a esta nueva universidad como una "institución moderna, con base científica, poniendo especial énfasis en la Investigación y la Extensión Universitaria" (1905:88). El propio Joaquín V. González definió en sus trabajos publicados en esos años a una universidad moderna, científicamente desarrollada, formadora de profesionales en los más amplios campos disciplinares, preocupada por el desarrollo social y cultural y comprometida con el progreso del país.

Esta corriente de pensamiento acerca de la universidad y su relación con el Estado y la sociedad se fue consolidando mediante expresiones, documentos y trabajos sostenidos por intelectuales, políticos y determinados sectores universitarios de aquellos años. Entre ellos estaba el movimiento estudiantil de la época, que venía expresando con mucha fuerza y claridad posiciones críticas y reclamos hacia las universidades existentes, impulsando propuestas y acciones para la transformación de las mismas. Uno de los momentos más significativos de dicho planteamiento quedó plasmado en el año 1908 en el marco del Congreso Internacional de Estudiantes Latinoamericanos, realizado en Montevideo. En ese Congreso, los representantes del movimiento estudiantil proveniente de la Argentina introdujeron de manera explícita a la extensión universitaria en una de sus acciones principales cuando plantearon: «Promover y difundir la Educación del pueblo, buscando el engrandecimiento nacional por medio de la educación de todas las clases sociales».

Si bien estos planteamientos y definiciones acerca de la universidad estaban presentes en toda esa época -incluso eran sostenidos con los primeros trabajos realizados hacia la comunidad-, fue sin lugar a dudas con la Reforma Universitaria de 1918 cuando se cristalizó un modelo de universidad que incorporó, entre otros aspectos, la función de extensión en toda su dimensión y con características propias como parte de la labor permanente de la institución universitaria.

Carlos Tünnermann Bernheim, consultor de Educación Superior de la UNESCO, dice:

"El primer cuestionamiento serio de la universidad latinoamericana tradicional tuvo lugar en 1918, año que tiene especial significación para nuestro continente, pues señala, según algunos sociólogos e historiadores, el momento del verdadero ingreso de América Latina el siglo XX. Las universidades latinoamericanas, como fiel reflejo de las estructuras sociales que la Independencia no logró modificar, seguían siendo los 'virreinatos del espíritu' y conservaban, en esencia, su carácter de academias señoriales. Eran, en realidad, 'coloniales fuera de la colonia'. La primera confrontación entre la sociedad, que comenzaba a experimentar cambios en su composición interna, y la universidad enquistada en esquemas obsoletos, se concretó en el llamado Movimiento o Reforma de Córdoba. Por supuesto que tal movimiento no puede ser examinado únicamente desde su ángulo académico universitario, por importantes que sean los cambios que en este campo se propiciaron. Necesariamente, es preciso considerarlo dentro del contexto socioeconómico y político del cual brotó. La clase media fue, en realidad, el protagonista clave del movimiento, en su afán por lograr acceso a la universidad, hasta entonces controlada por la vieja oligarquía terrateniente y el clero. La universidad aparecía, a los ojos de la nueva clase emergente, como el canal capaz de permitir su ascenso político y social. De ahí que el movimiento propugnara por derribar los muros anacrónicos que hacían de la universidad un coto cerrado de las clases superiores". (2002:8)

De esta manera, el programa de la Reforma desbordó los aspectos puramente docentes e incluyó toda una serie de planteamientos político-sociales que aparecen ya insinuados en el propio Manifiesto Liminar de los estudiantes cordobeses de 1918. Continúa Tünnermann Bernheim diciendo que:

"el fortalecimiento de la función social de la universidad, vía protección de su quehacer a la sociedad mediante los programas de Extensión Universitaria y Difusión Cultural, figuró desde muy temprano entre los postulados de la reforma de 1918. En realidad, la 'misión social' de la universidad constituía el remante programático de la reforma. De esta suerte, el movimiento agregó al tríptico misional clásico de la Universidad, un nuevo y prometedor cometido, capaz de vincularla más estrechamente con la sociedad y sus problemas, de volcarla hacia su pueblo, haciendo a este partícipe de su mensaje y transformándose en su conciencia cívica y social. Acorde con esta aspiración, la reforma incorporó la Extensión Universitaria y la Difusión Cultural entre las tareas normales de la universidad 
latinoamericana y propugnó por hacer de ella el centro por excelencia para el estudio científico y objetivo de los grandes problemas nacionales. Toda la gama de actividades que generó el ejercicio de esta misión social, que incluso se tradujo en determinados momentos en una mayor concientización y politización de los cuadros universitarios, contribuyeron a definir el perfil de la universidad latinoamericana, al asumir esta, o sus elementos componentes, tareas que no se proponían o que permanecían inéditas para las Universidades de otras regiones del mundo". (10)

Como se puede apreciar, la incorporación de la extensión universitaria y de la difusión cultural entre las tareas de la universidad latinoamericana forma parte de un proceso histórico encaminado a lograr una mayor apertura y democratización de la universidad y una más amplia proyección social de su labor. Los documentos del movimiento reformista, así como las opciones de sus teóricos, permiten notar el concepto que ellos tuvieron de este nuevo cometido universitario. En el Manifiesto Liminar del 21 de junio de 1918 los estudiantes denunciaron el "alejamiento olímpico" de la universidad, su total despreocupación por los problemas nacionales y su "inmovilidad senil", por lo mismo que era "fiel reflejo de una sociedad decadente". "Vincular la Universidad al Pueblo" fue así uno de los postulados de la Reforma que debía inspirar la tarea llamada de extramuros o de extensión universitaria. En este sentido, nuevamente Tünnermann Bernheim expresa que: "se pensaba que mediante este tipo de tareas el estudiante tendría la oportunidad no solo de familiarizarse con los problemas de este medio y de entrar en contacto con su pueblo, sino también la ocasión de devolver a este, en servicios, parte del beneficiario que significa permanecer a una minoría privilegiada que tenía acceso a una educación superior pagada en última instancia, por el esfuerzo de toda la comunidad. De la firme convicción de que la educación superior pública, financiada por el pueblo, debía revertir a él sus beneficios mediante las tareas de extensión llevadas a cabo por los estudiantes y sus profesores, surgió toda una serie de programas que tenían lugar en las fábricas, talleres y sedes sindicales, y cuya concreción más completa fueron las Ilamadas 'Universidades Populares' que se crearon, en el siglo XIX, en varios países europeos (Bélgica, Italia y Francia). En las Universidades Populares, cuyo personal docente estaba integrado fundamentalmente por estudiantes, confraternizaron estudiantes y obreros. De paso cabe señalar que Víctor Raúl Haya de la Torre reconocía que el APRA peruano surgió, precisamente, del contacto obrero-estudiantil que tuvo lugar en las 'Universidades Populares González-Prada', creadas por el reformismo peruano". (11)
Del Mazo, uno de los ideólogos del movimiento reformista, sostiene que:

"el afán de proyectar la labor universitaria en el seno de la colectividad, que fue uno de los enunciados básicos de la reforma, dio origen a una nueva función para la universidad latinoamericana, la función social, esto es, el propósito de poner el saber universitario al servicio de la sociedad y de hacer de sus problemas tema fundamental de sus preocupaciones. Precisamente, esta nueva función, que va más allá de las funciones clásicas atribuidas a la universidad, representan para varios teóricos de la Universidad Latinoamericana la que más contribuye a tipificarla y a distinguirla de sus congéneres de otras regiones del mundo". (1968:42)

Queda claro entonces que el movimiento reformista de 1918 impulsó un modelo de universidad totalmente diferente del existente por entonces en el país y sumamente novedoso a nivel mundial. Este modelo de universidad se caracterizó tanto por su definición respecto del concepto de la extensión asociada a la "función social de la universidad", a la "proyección al pueblo de la cultura universitaria" y a la "atención a los problemas nacionales", como por el resto de sus postulados que definían de manera integral a una universidad con autonomía (económica, administrativa, política y académica), gobernada democráticamente por su propia comunidad universitaria, con docencia libre, con ingreso irrestricto y asistencia social a los estudiantes, propiciando la creación de nuevas carreras y facultades e impulsando la unidad latinoamericana y a una formación cultural de los profesionales. En los tiempos actuales, la misión social de la universidad se resignifica a partir del valor del conocimiento y al entender a la propia educación como un derecho social y humano fundamental. En este sentido, es importante destacar la centralidad que tiene el conocimiento en cada una de las funciones sustantivas de la universidad. De acuerdo con la manera en que se transmite, circula, se crea o se apropia socialmente el conocimiento, estamos en presencia de las diferentes funciones sustantivas académicas de las instituciones universitarias. Por esta razón, la extensión universitaria adquiere una dimensión académica institucional sustantiva a partir de su íntima relación con el conocimiento, presente en cada una de sus prácticas. En cada acción de extensión, en sus prácticas, proyectos o programas, se ponen en juego conocimientos adquiridos y/o desarrollados por parte de la comunidad universitaria que dialogan con los conocimientos y saberes presentes en el medio sociocultural y socioproductivo en el que se interviene.

Desde este lugar, desde la educación y el conocimiento como acto democratizador para la transformación y desarrollo social, 
De acuerdo con la manera en que se transmite, circula, se crea o se apropia socialmente el conocimiento, estamos en presencia de las diferentes funciones sustantivas académicas de las instituciones universitarias

se resignifica el propio concepto de misión y compromiso social y cultural de la universidad, donde la extensión adquiere un rol central en cada práctica transformadora.

\section{Conceptualización y principales dimensiones de la extensión} Resulta importante remarcar que desde el modelo reformista del ' 18 se considera a la extensión como una de las tres funciones sustantivas que, en conjunto con la docencia y la investigación, constituyen los pilares básicos en los que se asienta el modelo universitario vigente en la mayoría de los países latinoamericanos. Así, cuando nos referimos a la extensión universitaria estamos considerando la relación interactiva que la universidad plantea con su medio en las dimensiones sociales, culturales y productivas, a partir de la circulación del conocimiento y en la búsqueda de la democratización y apropiación social del mismo, tal como se ha expresado anteriormente.

La extensión universitaria ha sido motivo de profundos análisis y debates históricos, signados por marcadas diferencias de enfoques teóricos conceptuales, que le han otorgado a esta función sustantiva características polisémicas y multidimensionales. Sin lugar a dudas, estos diferentes enfoques tienen una directa relación con los diversos modelos, tradiciones o corrientes ideológicas que se han ido construyendo acerca de la universidad en sus diferentes momentos históricos. La "universidad elitista", "profesionalista", "napoleónica", "reformista", "universidadempresa", "popular", "de los trabajadores", "militante", la "universidad para el desarrollo", son solo algunos de los tantos rótulos que reflejan la existencia de distintas posiciones o tradiciones acerca de universidad.

Estos modelos o posicionamientos referidos a la universidad han dado lugar a varias tipologías de extensión universitaria y han marcado sus principales políticas y acciones. Pero, además, la extensión universitaria ha estado en directa relación con el desarrollo académico, científico-tecnológico, histórico e institucional de cada universidad. Todo ello prefigura ciertas complejidades que envuelven a la extensión universitaria y la dificultad, muchas veces manifiesta, respecto de poder acordar definiciones, objetivos comunes y alcances en acciones y políticas. Esto ha estado presente en los muy diversos encuentros, jornadas, congresos, coloquios y simposios de alcance nacional, regional e internacional sobre extensión universitaria. Sin entrar a desarrollar, en el presente trabajo, los diferentes modelos de universidad, así como las diversas tipologías de extensión, se muestran a continuación las dimensiones más importantes ${ }^{1}$ que se encuentran presentes en la extensión universitaria y que le confieren una singular riqueza conceptual y han permitido su revalorización y resignificación en las últimas décadas en las universidades latinoamericanas y caribeñas:

Dimensión académico-institucional. Refiere a la extensión como una función sustantiva, integrada a la docencia y la investigación, en tanto que concibe al conocimiento como el elemento esencial presente en cada una de dichas funciones. La extensión presenta un rol central en su relación con el conocimiento. Es función esencial de la extensión promover la democratización y apropiación social del conocimiento, generar procesos de diálogo entre saberes y conocimientos y propender a la generación de cambios y transformaciones en su medio social, productivo y cultural, atendiendo de manera especial a los sectores más vulnerables y vulnerados socialmente.

En esta dimensión sustantiva, se plantea — cada vez con más fuerza en los últimos años- su institucionalización e incorporación curricular, su integración con la enseñanza e investigación, su jerarquización y reconocimiento académico, una asignación presupuestaria adecuada y el desarrollo de dispositivos de 


\section{G6 \\ la extensión universitaria es poseedora \\ de una dimensión pedagógica con \\ capacidad de contribuir de manera \\ significativa en los procesos de \\ enseñanza y aprendizaje.}

monitoreo, autoevaluación y reflexión críticas de sus prácticas. En importante además definir claramente los alcances que se reconocen a la extensión en esta dimensión sustantiva. La extensión universitaria aborda las más diversas problemáticas sociales, culturales y productivas donde la universidad interviene. La extensión universitaria promueve, a través de sus prácticas y acciones, una mejor calidad de vida, mayor inclusión y cohesión social y un desarrollo humano y sustentable. Desde este lugar, se impulsa una universidad comprometida socialmente e involucrada con el Estado en sus diferentes jurisdicciones, en las políticas públicas, abordando y sumando esfuerzos a la solución de problemáticas o al tratamiento de temáticas sociales, culturales o productivas. Desde allí se mira críticamente a la sociedad y al Estado y simultáneamente se interpela a la propia institución universitaria y se reflexiona sobre sus prácticas e intervenciones.

\section{Dimensión comunicacional en términos dialógicos. Esta} dimensión confiere a la extensión su propia esencia. Decimos que todo proyecto o trabajo de extensión, toda práctica o acción de intervención social, se da en términos dialógicos, de interacción y construcción mutua, lo que es precisamente la antítesis de invasión, manipulación, imposición o dominación. Significa sumar esfuerzos en la reflexión crítica y en cuanto a considerar a cada persona como verdadero sujeto de transformación.

En cada acción de extensión se pone en juego, en el medio social donde se desarrolla, los saberes y conocimientos de todos, se busca su problematización en su indiscutible relación con la realidad concreta en la cual dichos conocimientos o saberes circulan y/o se generan y sobre la cual inciden, para comprenderla mejor, explicarla y transformarla, y promover la reflexión crítica a la acción. Esta asociación de la extensión, como acción de comunicación en términos dialógicos y construcción mutua, está dada en el sentido planteado por Freire (1971) en su mirada crítica sobre la educación y la comunicación y en los desarrollos realizados por Habermas (1981) en su teoría de la acción comunicativa. Al identificar a toda acción de extensión como una acción comunicativa en términos dialógicos entre los sujetos participantes se asume que el contenido del conocimiento "extendido" circula en un espacio común en el que se considera que cada uno es sujeto del conocimiento y no mero receptor del mismo.

Desde esta perspectiva, es preciso ver a los actores interactuar con la realidad, y es en esa relación dialéctica que concebiremos un proceso de constante transformación y construcción, donde la relación universidad-sociedad es promotora de acciones transformadoras.

Esta dimensión dialógica remite además al indispensable "encuentro" inter y transdisciplinario que implica generar espacios de diálogo y profundas integraciones disciplinares a la hora de abordar las muchas y complejas problemáticas sociales.

\section{Dimensión social en términos de transformación. La} educación y el conocimiento para la transformación social (doble hermenéutica). Esta dimensión reconoce a la extensión en su acción transformadora. Toda práctica auténtica de extensión implica la generación de transformaciones en el medio social y en todos sus participantes al abordar las complejidades del medio 
social, cultural y productivo. Se encuentra íntimamente relacionada a la dimensión dialógica - expresada en el punto anterior- dado que todo proceso de transformación social requiere de la indispensable presencia de empatía, alteridad y entendimiento entre todos los participantes. Este necesario entendimiento entre los diferentes actores participantes nos introduce al concepto de doble hermenéutica y a los planteamientos realizados por Giddens (1984), así como al rol de la educación y del conocimiento científico en los procesos de transformación social.

En estos procesos de construcción social se pueden identificar diferentes ópticas desde las cuales son llevados a cabo. Uno de estos procesos se realiza desde los propios actores sociales involucrados, quienes - a través de acuerdos—interactúan y construyen sus propios objetivos; asimismo, se va trabajando sobre la percepción que ellos tienen de su situación actual, de sus fortalezas, debilidades, oportunidades y amenazas.

Un segundo proceso responde a la construcción de una visión de los problemas desde el "mundo técnico". Esta visión, de carácter más sistémico y disciplinar, reveladora de una lógica tanto cuantitativa como cualitativa, recorre el objeto de estudio e intervención a través de diversas miradas disciplinares, las cuales se integran y conforman una percepción técnica interdisciplinar que resulta complementaria de la construcción hecha por los actores sociales. Este doble proceso que se moviliza genera acciones que se retroalimentan mutuamente: validan la percepción social en algunos casos, reconocen dimensiones analíticas que emergen de la sociedad para ser indagadas desde lo técnico en otros, abren campos de dudas e incertidumbres que deben ser retomados para su análisis desde los sectores sociales involucrados, buscan causas explicativas (razones de fondo) de los problemas identificados por el grupo o la comunidad, señalan fortalezas, oportunidades, debilidades y amenazas que no son percibidas o reconocidas por parte de la sociedad, las cuales pasan a ser consideradas y evaluadas por la misma.

Esta doble hermenéutica supera las limitaciones clásicas de otros enfoques:

- El de una hermenéutica que solo se apoye en la visión desde el mundo técnico

- por la incapacidad de formular los objetivos sociales que serán capaces de movilizar a los actores con los que se trabaja; - porque implica desconocer que la decisión de un grupo, o de una comunidad de transformarse a sí misma le es inherente y por lo tanto solo puede ser asumida por el conjunto.

- El de una hermenéutica que solo se apoye en la percepción social (desde los actores)

- porque supone desconocer el valor del conocimiento técnicocientífico;

- porque implica desconocer también que los problemas sociales no solo están constituidos por aquellos factores que se hicieron explícitos, es decir desconocer lo latente, ignorar lo potencial; - porque es privar al proceso de construcción de consensos de las herramientas necesarias de validación, las cuales operan en muchos casos como factores catalizadores.

Este enfoque acerca de la comunicación entre actores y el diálogo entre los mismos, y entre los saberes y conocimientos científicos, nos interpela como universidad a realizar miradas críticas respecto de nuestras propias prácticas. En un trabajo o proyecto de extensión, o una investigación orientada o una práctica académica con sentido de intervención social: ¿con qué metodologías y enfoques de planificación trabajamos? ¿Lo llevamos adelante desde su formulación con los diferentes agentes involucrados? ¿Se buscan los suficientes consensos desde la identificación de los problemas a abordar hasta las acciones a realizar? ¿Nos involucramos en la realidad donde vamos a intervenir? ¿Se promueve una construcción interactiva y dialógica? ¿Qué lugar le damos al "otro" en nuestras prácticas? ¿Qué ponemos en juego en cada práctica? ¿Cómo nos atraviesan estas prácticas? Estos son algunos de los tantos interrogantes que deberíamos responder a la hora de emprender una acción de intervención social.

Dimensión pedagógica. Se reconoce, además, que la extensión universitaria es poseedora de una dimensión pedagógica con capacidad de contribuir de manera significativa en los procesos de enseñanza y aprendizaje. A partir de esta dimensión surge la propuesta de incorporar las prácticas de extensión en el currículo, planteado desde una perspectiva diferente de otros paradigmas pedagógicos. Es una invitación a pensar que el conocimiento será más significativo y profundo si tiene origen en los contextos mismos donde los saberes se producen. Las ideas de John Dewey acerca de la importancia de la experiencia, su lugar y su sentido en las instancias de formación, se entrelazan con nuevas ideas en el campo de la educación que hablan de la importancia de los aprendizajes y actividades situadas; ambas intentan justificar esta propuesta. Para los teóricos de la actividad situada, el aprendizaje es el proceso mismo que se da en las relaciones que se tejen entre personas, actividades y situaciones como una sola entidad abarcadora. Actividad, aprendizaje y contexto, pensamiento, sentimiento y acción son dimensiones que se entrelazan en un todo unificado.

Así, entender la educación, conocer y aprender implica comprometerse en un mismo proceso cambiante, difícil e incierto, donde el conocimiento y el aprendizaje se constituyen en campos también complejos y problemáticos. El conocimiento transcurre dentro de los sistemas de actividad que se desenvuelven social, cultural e históricamente, e involucra a personas que se vinculan de manera múltiple y heterogénea. En el recorrido dado, en 
cómo se aprende, es posible mostrar que se pueden crear nuevos conocimientos interviniendo en la práctica, en la actividad misma; de allí la necesidad de participar en escenarios sociales amplios. En este sentido, se demuestra que puede existir un potencial educativo muy grande al momento de intervenir desde la educación formal en prácticas sociales concretas, donde el conocimiento circula, se transforma, se crea y recrea.

Una propuesta de esta naturaleza recupera la importancia del intercambio social en la construcción de conocimientos. Desde la dimensión pedagógica, incorporar la extensión al currículo implica una innovación; por lo tanto, es posible que por momentos tenga que competir, reemplazar, modificar, o incluso integrar aquellas teorías ya formadas sobre las maneras de concebir la formación universitaria tanto para docentes como para estudiantes universitarios.

La búsqueda de estrategias didácticas posibilitadoras de la construcción y elaboración de nuevos aprendizajes lleva a plantear como alternativa posible la enseñanza a partir de las prácticas sociales (asociadas a las prácticas de extensión), que se constituyen en prácticas académicas en las que "se aprende en situación". Creemos que es una razón de peso considerar a las prácticas de extensión como un valioso recurso pedagógico para promover una "actitud" crítica y comprometida en cuanto al rol que como profesional puede tener en la democratización del conocimiento y la generación de cambios sociales innovadores.

Esta orientación metodológica o modalidad educativa podría colaborar en el tratamiento crítico de aspectos que forman parte del "curriculum oculto", es decir, de las prácticas cotidianas que, sin ser explicitadas, existen y se concretan dentro o fuera del aula. Nos referimos a las experiencias antes descriptas y que no son totalmente ajenas en el ámbito de la enseñanza universitaria: la transmisión del conocimiento como una "entidad" inalterable que produce, entre otras, una visión fragmentaria y acrítica sobre el mismo, ocultando aquella esencia de un "proceso" en el que el estudiante puede y debe jugar un rol importante en su construcción y reconstrucción.

Los estudiantes deben disponer de una gran variedad de oportunidades y materiales para promover el aprendizaje "profundo", ofrecer suficiente evidencia en la que basar el conocimiento y, mediante la reconstrucción de las vivencias, reflexionar acerca de su propio desempeño.

El papel de la extensión no solo contribuirá a identificar problemas sobre los que se puedan intervenir, o bien promover estudios e investigaciones sobre los mismos, sino que puede contribuir a poner a prueba, a validar las soluciones —-teóricas, técnicas- producidas.
El abordaje de los dilemas éticos vinculados a toda profesión puede ser estimulado a partir de la participación de los estudiantes en este tipo de prácticas, ya que al verse en situaciones concretas cuentan con una oportunidad para conocer, asumir y reflexionar sobre las responsabilidades propias de la profesión.

Además, este es un espacio propicio para el desarrollo de actitudes y valores, para poner en práctica los conceptos de alteridad y empatía que motiven el compromiso con otros y la iniciativa, preparándose para adaptarse a los cambios y ser partícipes directos en tales cambios.

Se rescata, asimismo, el trabajo interdisciplinario que compromete no solo la cooperación entre las disciplinas presentes en la formación sino, esencialmente, la construcción colectiva y consensual del proyecto de formación, universidad-sociedad, lo que posibilita el aprendizaje desde y en múltiples direcciones.

En definitiva, la incorporación curricular de este tipo de prácticas de extensión, a las que denominamos Prácticas de Educación Experiencial $^{2}$ —siguiendo a David Kolb (1984)—, supone para los estudiantes y docentes experimentar un proceso de aprendizaje en situación de una intervención en el medio social y abordar problemas concretos, en tanto que la educación experiencial es

"una forma de aprendizaje a través de la experiencia en el que los estudiantes se involucran en actividades que atienden necesidades humanas y comunitarias, junto con oportunidades de aprendizaje estructuradas e intencionalmente diseñadas para promover el aprendizaje de los estudiantes y su desarrollo. Reflexión y reciprocidad son conceptos claves de esta modalidad". (Jacoby, 1996:5)

Dimensión política. Finalmente, se reconoce también en la extensión la existencia de una dimensión política que implica el involucramiento de la universidad con la sociedad y el Estado de los cuales aquella forma parte. No existe programa, proyecto o acción de extensión que no se realice en términos de intervención en espacios sociales. Desde este lugar, la universidad se relaciona con la "cosa pública". Se expresa, actúa, reflexiona, construye con el "otro", brinda sus conocimientos y también adquiere aprendizajes a partir de cada práctica que realiza.

Tal como se ha visto en el desarrollo de este trabajo, desde la extensión, toda intervención plantea una intención o interés de cambio o transformación en el medio social en el que se interactúa. Esta dimensión política de la extensión nos lleva a pensar el vínculo de la universidad con el Estado y la sociedad en términos de políticas públicas. ${ }^{3}$ Uno de los temas centrales de la agenda de extensión, y con ella de la propia universidad, está vinculado a
2) Para ver el desarrollo de las Prácticas de Extensión de Educación Experiencial en la Universidad Nacional del Litoral consultar: Menéndez y otros
(2013); Menéndez y Tarabella (2016); Boffelli y Sordo (2016) y Menéndez y otros (2017).

3) Ver Menéndez (2012). 
las políticas públicas, espacios de intervención y de construcción colectiva en su desarrollo, calidad, alcance y ejecución. Esta aproximación a las políticas públicas tiene como punto de partida la definición del rol que las universidades adoptan en relación con el Estado en sus diferentes jurisdicciones y la sociedad de la cual forman parte.

Entendemos a las políticas públicas como las acciones que el Estado realiza en un contexto histórico en particular, y que sin duda remiten al modelo de acumulación vigente como también al desarrollo de las políticas a nivel global. Reflejan tanto los vínculos existentes entre los habitantes de un territorio y las autoridades del poder público como los mecanismos de distribución del poder. A su vez, constituyen espacios de interacción social, donde se definen intercambios entre sujetos que participan en un campo que, desde la óptica bourdesiana, está compuesto por relaciones históricas objetivas, entre posiciones que desnudan ciertas formas de poder o capital.

Desde este lugar, podemos afirmar que las universidades son parte de esas políticas públicas y, a la vez, son instituciones sociales y agentes de transformación. Al poseer un capital simbólico y cultural importante, la universidad obtiene legitimidad en aquellos espacios en donde el conocimiento se pone en juego. Al consagrarse particularmente el conocimiento científico como un vector de poder significativo para el mundo contemporáneo, las convierte en un espacio estratégico y en un interlocutor insoslayable a la hora de pensar en políticas de Estado.

A su vez, esta dimensión política de la extensión nos introduce al campo de la ética entendida como un conjunto de principios y valores que determinan la conducta del individuo como un ser social. La ética se constituye en un factor central en los modelos de sociedades y en la formación de diversas visiones en los ámbitos que la componen: político, económico, social y cultural. En este sentido, las universidades públicas tienen un rol central a través de la educación y en la apropiación social de los conocimientos, en la reproducción social de principios y valores que sostienen y alimentan una concepción ética presente en la vida de las personas y en las instituciones.

\section{Entramado de categorías teóricas vinculadas a la extensión universitaria}

Al momento de definir las políticas de extensión y su relación con las políticas públicas, es indispensable identificar las principales categorías teóricas que deberían ser tenidas en cuenta para el diseño de dichas políticas institucionales.

En primer lugar, no podemos dejar de realizar una mirada reflexiva acerca de la educación. Tanto las conferencias mundiales y regionales de la educación superior como los diversos congresos y encuentros de educación han sido escenarios en los que han estado presentes diferentes enfoques y concepciones respecto de la educación y sus aportes al desarrollo de la sociedad y de las naciones. No es lo mismo desarrollar políticas desde las universidades desde una visión sociocrítica, entendiendo a la educación como un derecho social y humano fundamental, que hacerlo considerando a la educación como un servicio que se rige por las leyes del mercado. Las prácticas de extensión (programas, proyectos, acciones) cambiarán significativamente si entendemos a la educación como un derecho y un acto político de transformación o si entendemos a la educación como un servicio que se compra o se vende en un mercado.

Por otra parte, aparece la universidad como objeto de estudio, en el que la autonomía, pensamiento crítico, democracia, pertinencia y calidad se encuentran interactuando y retroalimentándose en una muy sensible y delicada tensión permanente. Aquí también, 


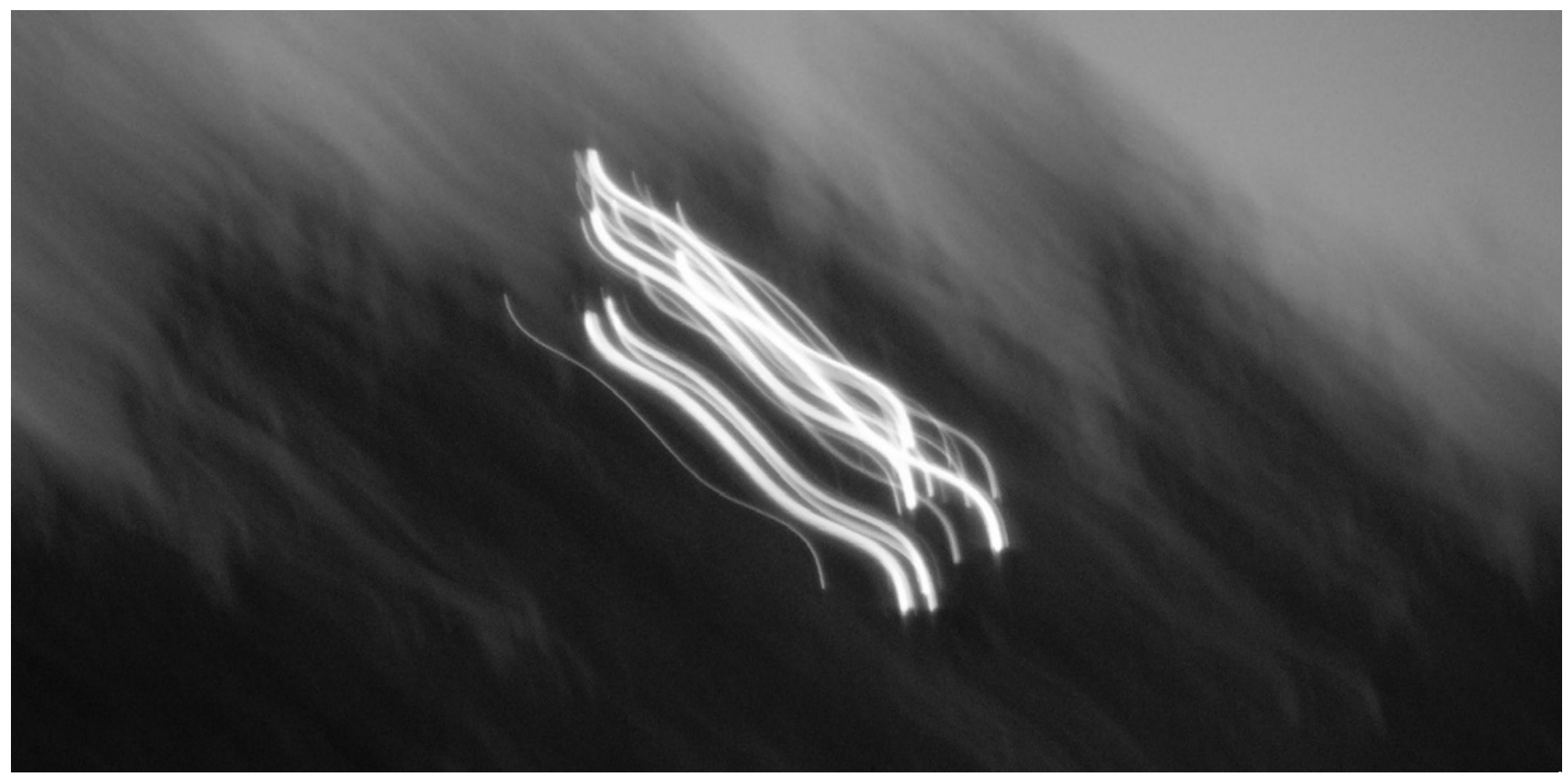

(C) Laura Malachesky

una práctica de extensión será diferente si se lleva adelante desde un modelo de universidad donde se promueve el desarrollo del pensamiento crítico y la autonomía, donde se ponen en ejercicio permanente la construcción democrática y la ciudadanía, donde además se realiza en una búsqueda permanente de la calidad, que si esta práctica de extensión se realiza desde otro modelo o concepción de universidad.

Asimismo, conocimiento y poder son categorías teóricas que no podemos soslayar cuando se formulan propuestas institucionales que planteen la participación de la universidad en el medio social, cultural y productivo en el que intervendrá. A la hora de realizar una práctica de extensión es importante profundizar la mirada conceptual acerca del conocimiento y del poder, ya que no existe práctica de extensión en la que no se pongan en juego conocimientos adquiridos y/o desarrollados por la universidad; así también, en toda intervención en la que se constituye una práctica de extensión existe una intención de cambio o transformación social al abordar problemáticas culturales, sociales o productivas. Este proceso de transformación se realiza en un espacio microsocial donde existe un permanente juego de poder entrelazado con intereses, sentimientos y anhelos de todos y cada uno de los participantes.

En este aspecto, se reconoce que el conocimiento es el "elemento" común, presente en cada una de las funciones sustantivas de docencia, extensión e investigación. Tal como se expresó anteriormente, es el conocimiento el que está presente en los procesos de formación, es el conocimiento científico y también social el que se pone en juego en cada programa, proyecto o práctica de extensión, y es el nuevo conocimiento a ser generado el que se plantea desde las políticas científicas. Y es precisamente desde la relación del conocimiento y poder donde surgen preguntas tales como: ¿de qué manera se genera y circula el conocimiento? ¿Para qué y para quién es dicho conocimiento? ¿Se traduce en las políticas institucionales la efectiva democratización de los conocimientos? ¿Cuáles son los problemas centrales a abordar? ¿Qué intereses, conflictos y potencialidades se encuentran presentes? ¿Cómo se da la relación entre conocimiento y poder? ¿De qué manera se dan los procesos de transformaciones sociales? ¿Quiénes se constituyen en aliados en los procesos de intervención? Estos son algunos de los tantos interrogantes que aparecen cuando se formulan políticas de extensión y que deben ser tenidos en cuenta ya que las respuestas a los mismos determinan las direcciones que van a tener dichas políticas.

Por otra parte, cuando hablamos de extensión y su vínculo con las políticas públicas no podemos dejar de analizar de manera muy profunda la sociedad en la que vivimos, sus paradigmas y complejidades, ya que toda acción de extensión se vincula directamente con actores concretos, en territorios definidos, y aborda las más diversas problemáticas existentes. Por ese, la sociedad, los espacios sociales y el territorio se constituyen en categorías centrales que debemos tener en cuenta cuando se realiza una intervención desde un proyecto, programa o acción de extensión. 
Ya hemos visto, además, la relación existente entre comunicación, extensión y educación en términos dialógicos al momento de formular y llevar adelante una acción de extensión. No existe práctica de extensión en la que no se intervenga de manera interactiva y dialógica con el medio social con el que se relaciona. Aun más, el proyecto, programa o acción de extensión comienza a partir del vínculo que se establece en términos de diálogo y acuerdos. En este entramado de categorías teóricas, aparecen también los términos de alteridad y empatía a la hora de definir propuestas de extensión: ¿qué lugar tiene "el otro" en cada una de nuestras prácticas? ¿Cómo veo a ese "otro" y qué opinión percibo que tiene ese "otro" de mí? ¿Considero que su palabra es tan importante como la mía? En toda práctica de extensión, la empatía y la alteridad son elementos esenciales e imprescindibles que representan la capacidad de desarrollar vínculos con el "otro", reconocer a ese "otro" tal cual es y poder crecer juntos en las diferencias.

La visión acerca del desarrollo humano sustentable, en términos de inclusión y cohesión social, constituye una categoría teórica esencial que se transforma en el objetivo central hacia el cual van dirigidos los esfuerzos de la universidad a través de la extensión universitaria.

Cada acción, trabajo o proyecto de extensión tiende a realizar un aporte concreto al fortalecimiento de la democracia, la ciudadanía y las políticas públicas, las que también se constituyen en categorías teóricas centrales al momento de formular y desarrollar políticas de extensión.
Entendiendo a la extensión en sus diferentes dimensiones y teniendo en cuenta las categorías teóricas antes presentadas, se identifica un conjunto de objetivos generales que da lugar a los programas y proyectos institucionales más importantes que han venido construyendo las universidades públicas latinoamericanas y caribeñas a partir de asumir plenamente su compromiso para con la sociedad a través de sus políticas institucionales.

\section{¿Cómo se proyecta la extensión universitaria para los próximos años?}

En estudios realizados sobre la extensión en las universidades latinoamericanas y caribeñas, se detecta un conjunto de objetivos en sus políticas institucionales e instrumentos de gestión que marcan una agenda. De ellos, se destacan los siguientes:

- Institucionalización y reconocimiento académico de la extensión.

- Integración de la extensión con la docencia y la investigación.

- Democratización del saber y del conocimiento.

- Búsqueda de la apropiación social del conocimiento y generación de nuevos conocimientos socialmente acordados.

- Formación de ciudadanos críticos y comprometidos socialmente. - Aportes hacia una sociedad más inclusiva, democrática, justa y solidaria.

- Compromiso para con las políticas públicas y en la construcción de un modelo de desarrollo humano sustentable.

- Profundización en su misión social y cultural. 
A partir de lo expuesto hasta aquí respecto del significado de la misión social de la universidad reformista, de las dimensiones y categorías teóricas más importantes presentes en la extensión universitaria a ser tenidas en cuenta a la hora de realizar una práctica de extensión, y considerando los objetivos más importantes que las universidades de la región vienen planteando en sus políticas e instrumentos de gestión en materia de extensión, se presentan a continuación los aspectos más relevantes que podrán ser considerados en el estudio, monitoreo, gestión, formación e investigación de la extensión universitaria.

Estos aspectos se vienen incorporando en las políticas institucionales, en los planes de desarrollo institucional, en las normativas e incluso en los propios estatutos que las comunidades universitarias están consensuando en materia de extensión universitaria.

De manera enunciativa, estos son algunos de los ejes más importantes que forman parte de las políticas institucionales y de sus agendas de trabajo:

- Institucionalización y reconocimiento académico de la extensión.

- Integración de la extensión con la docencia y la investigación.

- Fortalecimiento institucional de los sistemas o programas institucionales de programas y proyectos de extensión.

- Acción territorial de la extensión, intervención en los espacios microsociales.

- Análisis y aportes a las políticas públicas.

- Internacionalización de la extensión.

- Formación y capacitación en extensión.

- Desarrollo institucional de la editorial.

- Programas culturales.

- Programas socioproductivos de apropiación social de los conocimientos y de innovación.

- Planeamiento y evaluación de la extensión.

- Programa de comunicación de la extensión en el campo de la "comunicación-educación-extensión".

- Programa presupuestario y asignación de recursos.

- Sistema de gestión administrativa.

- Desarrollo de capacidades de gestión y equipos de trabajo.

\section{Algunas reflexiones finales}

Es importante mencionar que la identificación de estos ejes prioritarios de trabajo ha permitido analizar de manera crítica el desarrollo de las políticas, instrumentos de gestión, acciones y resultados de la extensión en los últimos 15 años de gran parte de las universidades latinoamericanas y caribeñas. El reconocimiento de la riqueza conceptual de la extensión, su potencial rol de transformación social y su contribución al desarrollo institucional ha hecho posible que la extensión universitaria hoy forme parte significativa de la vida académica de las universidades, integrada con la docencia y la investigación, participe en los planes de desarrollo institucional en diversas universidades de la región y en especial aquellas que se identifican con el modelo de universidad reformista. En este sentido, se destacan las propuestas que vienen llevando adelante las redes de extensión pertenecientes a los sistemas universitarios en varios países de Latinoamérica y el Caribe mediante la formulación de planes de desarrollo de la extensión, sobre la base de ejes prioritarios de trabajo como los que se mencionan en el capítulo anterior.

Estos planes cuentan con diferentes mecanismos e instrumentos de gestión, entre los que podemos mencionar: programas o sistemas integrados de programas y proyectos de extensión; programas de vinculación tecnológica, de desarrollo socioproductivo y de servicios, culturales, de formación en extensión universitaria, de incorporación curricular de la extensión, de investigación orientados a problemas sociales y productivos, de apropiación social de los conocimientos e innovación; observatorios universitarios y cátedras libres o abiertas; centros comunitarios; programas de voluntariado o compromiso social universitario; desarrollo de editoriales universitarias; políticas de internacionalización de la extensión, entre otros.

Desde esta visión y con el trabajo realizado, se resignifica el sentido de la pertinencia de la universidad, su función y compromiso social; se profundizan los objetivos planteados en el vínculo de la universidad con el Estado y la sociedad, en el que no solo se intenta contribuir al bienestar y a la calidad de vida de la población —en especial aquellos sectores en situación de vulnerabilidad social一, sino que además se plantea esta relación con base en una profunda necesidad académica que enriquece las líneas de investigación, las propuestas curriculares de grado y posgrado y la generación de nuevas líneas de extensión.

A partir del diseño y desarrollo de estas políticas institucionales y de las experiencias realizadas, las universidades de la región consideran cada vez más a la extensión como parte del proceso dinámico de la acción universitaria frente al conocimiento, en el cual se identifica a la sociedad y al Estado en sus diferentes jurisdicciones como fuente de saber y como interlocutores indispensables, que permiten interpelar el conocimiento científico y generar condiciones de diálogo con los saberes locales, de modo de lograr una mutua imbricación y un mutuo aprendizaje que enriquece tanto a las ciencias como a las comunidades. Fortalecer y profundizar las políticas y prácticas de extensión en las universidades en la dirección planteada en el presente trabajo implican el desarrollo integral de la propia institución universitaria, ya que resignifican el sentido de la misión social, interpelan a la 
docencia a pensar en otras formas de enseñar y de aprender, e invitan a la investigación a imaginar nuevas formas de construcción de conocimientos y la apropiación social de los mismos. Pero, para ello, se requiere construir espacios colectivos para afrontar en conjunto estos nuevos desafíos.

A casi 100 años de la Reforma Universitaria de 1918, sus principios fundamentales se mantienen con sorprendente vigencia en la búsqueda de una universidad democrática y democratizadora, con calidad y compromiso social, autónoma y con plena capacidad de desarrollar el pensamiento crítico. Esta universidad reformista hoy renueva su compromiso de pensarse a sí misma para afrontar los desafíos futuros en sociedades cada vez más complejas, desiguales e injustas. Y es aquí donde aparece como indispensable el rol de la extensión universitaria como función sustantiva, académica e institucional, con capacidad de interpelar e integrarse con la docencia y la investigación en la construcción de una sociedad más inclusiva, con pleno ejercicio de los derechos humanos, de la democracia, del sentido ético de la política, de la ciudadanía, de la solidaridad y del bien común. De esta forma, decimos que el ideario de la universidad reformista que se proyecta al futuro tendrá la capacidad de afrontar los desafíos que los tiempos le demanden si logra desarrollar las capacidades y fortalezas académicas, políticas e institucionales para la construcción de un mundo mejor. Si así fuera, podríamos decir con plena seguridad que esta universidad reformista del siglo XXI estaría en condiciones de asumir este legado histórico para dar respuesta a las diversas y complejas demandas planteadas por el Estado y la sociedad de los cuales forma parte y cumplir con el profundo reclamo que nos transmitía el Manifiesto Liminar de 1918, cuando señalaba que "los dolores que nos quedan son las libertades que nos falta conquistar".

\section{Referencias bibliográficas}

Boffelli, M. y Sordo, S. (2016). Fundamentos y perspectivas desde donde pensar la integración de la docencia y la extensión. Revista +E versión digital, (6), 16-23. Santa Fe: Ediciones UNL

Del Mazo, G. (1968). La Reforma Universitaria. Tomos I, II, y III. Lima: Universidad de Lima.

Freire, P. (1971). ¿Extensión o comunicación?

Giddens, A. (1984). La constitución de la sociedad: bases para la teoría de la estructuración. California: University of California Press.

González, J. V. (1905). La Universidad Nueva. La Plata: Universidad Nacional de la Plata.

Jacoby, B. (1996). Service-Learning in Higher Education: Concepts and Prac-

tices. Jossey-Bass.

Habermas, J. (1987). Teoría de la Acción Comunicativa. 2 vols. Madrid: Taurus.

Kolb, D. (1984). Experiential Learning. Englewood Cliffs, N. J. Prentice-Hall.

Menéndez, G. (2011). Desarrollo y conceptualización de la extensión: desafíos presentes y futuros. Revista $+E$, (1), 22-31. Santa Fe: Ediciones UNL.

(2012). Extensión y políticas públicas: la universidad en el centro del debate. Revista +E versión digital, (22), 40-46. Santa Fe: Ediciones UNL.

(2015). La Extensión Universitaria: Aportes para la construcción de la red de observatorios y cátedras abiertas y/o Libres. Ciudad de Puyo, Ecuador. Unión Latinoamericana de Extensión Universitaria (ULEU).

Menéndez, G. y otros (2013). Integración docencia y extensión. Otra forma de enseñar y de aprender, 1 y 2. Santa Fe: Ediciones UNL.

Menéndez, G. y Tarabella, L. (2016). El aprendizaje experiencial: una práctica de innovación que se afianza en la Universidad Nacional del Litoral. Revista + E versión digital, (6), 96-103. Santa Fe: Ediciones UNL.

Palacios Moroni, L. (2003). La extensión universitaria. Valencia: Universidad de Valencia.

Tünnermann Bernheim, C. (2002). El nuevo concepto de la Extensión Universitaria. Cuaderno $N^{\circ}$ 5. Venezuela: Universidad de Carabobo. 